\title{
Die Zeugung im Rausche.
}

\section{Mitteilung.}

\author{
Von
}

Ober-Med.-Rat Prof. Dr. P. Näcke, Colditz i. S,

(Eingegangen am 4. Mai 1913.)

Im Jahre 1908 hatte ich über dies Thema meine I. Mitteilung gebracht'1), nachdem die Zeugung im Rausche ein längst beliebtes Schlagwort geworden war und fast überall kritiklos hingenommen wurde. Ich hatte es wohl mit zuerst, sicher aber am eingehendsten unter die Lupe genommen und gezeigt, welche wissenschaftlichen Anforderungen hier bezüglich eines Kausalnexus gestellt werden müßten. Ist es schon in concreto oft nicht leicht, den Rausch während des Coitus nachzuweisen, so ist es mit dem Faktum der Zeugung noch viel schwieriger bestellt. Meist bleibt es ja nicht bei einem einzigen Beischlafe, sondern andere sind ihm kurz vorangegangen oder folgten $\mathrm{ihm}$. Welcher war dann der befruchtende? Es muß also zunächst nur ein einziger Coitus mit einem bestimmten Manne in trunkenem Zustande vorliegen. Im ehelichen Verkehr ist es leichter, hierüber Genaueres zu erfahren als im außerehelichen. Am schwierigsten bleibt es aber, den Zusammenhang zwischen Rauschzeugung und Minderwertigkeit des Geborenen aufzudecken. Es gilt hier vor allem zunächst einmal eine Krankheit oder Minderwertigkeit des Mannes und der Frau zur Zeit des Beischlafs sicher auszuschließen, um dem Rausch allein den eventuellen Schaden in die Schuhe zu schieben, namentlich aber die Trunksucht. Es könnte jedoch weiter noch eine latente erworbene oder an- resp. eingeborene Anlage, für welche die hereditäre Belastung einen gewissen Index darstellt, vorliegen, und auch dieser müßte man nachspüren.

Aber mit den Schwierigkeiten sind wir noch nicht zu Ende. Die Minderwertigkeit bzw. Entartung eines Kindes gibt sich nämlich gewöhnlich nicht gleich nach der Geburt zu erkennen, sondern erst allmählich, oft genug deutlich erst um die Zeit der Pubertät oder gar noch später. Man denke z. B. an die meisten Psychosen und viele Nervenkrankheiten. Wer will dann nach so langer Zeit sich noch sicher der Umstände erinnern, die die Zeugung des Betreffenden begleiteten, wenn

1) Näcke, Die Zeugung im Rausche und ihre schädlichen Folgen für die Nachkommenschaft. Neurol. Centralbl. 1908, Nr. 22. 
nicht etwa frappante Krankheiten oder besondere Umstände das Gedächtnis schärften? In der Ehe und bei Gebildeten läßt sich hierin noch am meisten erfahren. Aber wie viele könnten auch hier mit absoluter Sicherheit behaupten, daß es nur bei einem einzigen Coitus geblieben ist? Dabei ist die incertitudo patris noch gar nicht in Betracht gezogen. Eher läßt sich noch erinnern, ob man zur fraglichen Zeit bezecht war oder nicht. Ob man damals krank war oder schwere Sorgen hatte usw., läßt sich chronologisch oft genug nicht mehr feststellen. Kurz, man sieht, wie wenig man sich hier auch auf ein sog. gutes Gedächtnis verlassen kann. Und von latenter Krankheitsanlage und hereditären Belastungsmomenten wissen die wenigsten Genaueres zu berichten.

Man versteht also, daß ich bis 1908 keinen einzigen Fall kannte, der diesen strengen, aber selbstverständlichen wissenschaftlichen Anforderungen gegenüber standgehalten hätte. Ausdrücklich hatte ich aber schon in der ersten Publikation die Möglichkeit eines solchen Zusammenhanges zugegeben und gesagt: „Natürlich will ich nicht die Unmöglichkeit eines solchen Zusammenhanges behaupten, nur die außerordentliche Schwierigkeit des Nachweises und wahrscheinlich auch Seltenheit desselben." Und weiter: „Ist aber einmal ein wirklicher Zusammenhang zwischen Rausch und Zeugung minderwertiger Nachkommen festgelegt oder mindestens sehr wahrscheinlich gemacht, dann würde es uns weniger schwer fallen, die verschiedenen Arten der Entartung der Zeugungsprodukte uns vorzustellen...."

Es ließ sich nun voraussehen, daß Entgegnungen hierauf, besonders seitens der Abstinenzler - dieser Ausdruck ist z. Z. noch gebräuchlicher als: Abstinenten - erfolgen würden. So erschien 1909 eine solche zuerst von Holitscher ${ }^{1}$ ), an die ich speziell meine zweite Arbeit über den Gegenstand anknüpfte ${ }^{2}$ ). Holitscher konnte nur drei Beobachtungen sehr summarischer Art aus seiner Praxis anführen, die zwar nicht den gestellten wissenschaftlichen Anforderungen ganz entsprachen, immerhin aber praktisch gelten konnten. Entschieden wies ich dagegen hier die Massenuntersuchungen Bezzolas, Bayerthals usw. als Beweismaterial zurück und das $u$. a. wegen ihrer Vieldeutigkeit. „Sie können uns, “ sagte ich, „nur gewisse Hinweise geben, eine Möglichkeit eines solchen Zusammenhangs, mehr aber nicht." Es könne uns „nur eine große Sammlung einzelner genau beobachteter Fälle .... hier in der Erkenntnis weiter bringen." Ich schloß diesen zweiten Aufsatz mit den Worten, daß ,wahrscheinlich nur in sehr großen Ausnahmefällen die Rauschzeugung schädlichen Einfluß hat. Auf alle Fälle ist

1) Internat. Monatsschr. z. Erforschung d. Alkoholismus usw. Juli 1909.

2) Näcke, Zwei sexologische Themen: 1. Die Zeugung im Rausche und ihre schädlichen Folgen usw. Zeitschr. f. d. ges. Neur. u. Psych. 11, H. 1/2. 
aber nur eine Wahrscheinlichkeit für einen solchen Zusammenhang gegeben, nie und nimmer eine Sicherheit. Doch für praktische Zwecke genügt schon jene...."

Holitscher hat sich aber nicht beruhigt, sondern auch auf meine zweite Arbeit geantwortet ${ }^{1}$ ), was mir zu einigen allgemeinen Bemerkungen Anlaß gibt, noch mehr aber ein kurzer Aufsatz von Fels ${ }^{2}$ ), der zuletzt sich sogar bemüht, geistreich zu werden. Holitscher gibt selbst zu, daß seine 3 Fälle, die ich aber immer hier noch gelten lasse, nicht strengen wissenschaftlichen Forderungen gerecht würden, und er ist auch jetzt leider nicht in der Lage, noch weitere Beispiele anzuführen, was für die Seltenheit der Sache wohl sprechen dürfte. Meine Kritik jener 3 Fälle hält Fels, gewiß mit Unrecht, für zu weitgehend. Holitscher gibt auch zu, daß die Massenuntersuchungen Bezzolas wenig beweisend sind. Falsch ist dagegen seine Behauptung, ich hätte 1908 in meiner ersten Arbeit einen schroff ablehnenden Standpunkt bezüglich der Rauschzeugung eingenommen. Meine daraus oben zitierten Sätze beweisen dies hinreichend, nur daß ich jetzt die Sache etwas weniger ,schroff" hinstelle.

Fels fragt, ob denn geringe Unpäßlichkeiten, Schreck, Sorge usw. wirklich auf den Keim schädlich einwirken könnten. Natürlich gibt es hierüber keine Experimente ${ }^{3}$ ), aber wohl klinische Erfahrungen verschiedener Art, ähnlich wie nach schweren Erkrankungen, Vergiftungen usw. Bei geringen Unpäßlichkeiten allerdings ist ein solcher Zusammenhang wenig wahrscheinlich, das gebe ich zu. Eher schon bei länger andauernden, die den Patienten noch nicht zum Arzte führen, wie z. B. anämische Zustände, Zeiten von Schlaflosigkeit, leichte Genitalleiden. Zum Arzte geht der Kranke - wenn er nicht Kassenmitglied ist doch nur in ernsteren Fällen, wenn die Hausmittel nicht mehr ausreichen oder die Sache sich verschlimmert. Jeder, der praktiziert hat, weiß, was für verschleppte, alte Fälle er manchmal zu sehen bekommt, die noch nicht behandelt wurden. Anders ist es schon beim pauschaliter bezahlten Hausarzt, der leider immer seltener sich findet, dann aber wie der Kassenarzt bei jeder Kleinigkeit konsultiert wird.

Auf festerem Boden stehen wir bezüglich der Sorgen und des Schrecks. Wir wissen schon hinreichend, wie tief körperlich dieselben angreifen

1) Holitscher, Die Zeugung im Rausche. Internat. Monatsschr. z. Erforsch. d. Alkoholismus usw. Febr. 1913.

2) Einige Bemerkungen zu der Arbeit von Näcke usw. Zeitschr. f. d. ges. Neur. u. Psych. 11, H. 4 5. S. 647.

3) Bezüglich Schrecks, Furcht, Übermüdung usw. ließen sich wohl auch bei Tieren Experimente anstellen. Man setze z. B. in der Brunstzeit eine Hündin durch irgendwelche Manipulationen in Schrecken, der einige Zeit anhält und lasse sie dann belegen. Oder man lasse sie einige Stunden in der Tretmühle arbeiten bis zum Hinfallen und dann belegen. 
können. Die Neurologie und Psychiatrie liefern uns andererseits fast täglich Beispiele für ihr starkes Einwirken. Ja, die Rolle namentlich der lange andauernden depressiven Affektzustände wird jetzt besonders in der Ätiologie der Psychosen wohl mit Recht immer höher eingeschätzt. Wir wissen ferner, daß während solcher Zustände empfangene oder geborene Kinder sehr oft irgendwie minderwertig sind oder es werden. Von diesen Sorgen, Kümmernissen usw. erfährt der gelegentlich befragte Arzt oft genug gar nichts, eher schon der Hausarzt. Letzter wird nun wohl über die körperliche und psychische Konstitution seiner Patienten, wenn er sie oft sieht, urteilen können, anders dagegen, wenn es nur selten geschieht. Aber auch im ersten Falle wird ihm gewöhnlich eine eingeborene latente Krankheitsanlage, wie sie sich namentlich in der erblichen Belastung zeigt, entgehen. Ich habe selbst jahrelang praktiziert und war in vielen Familien Hausarzt. Uber die etwaige erbliche Belastung der Kranken habe ich fast nie etwas gewußt und zwar aus dem einfachen Grunde, weil kein Anlaß dazu vorlag. Wo es sich um Phthise, Gicht, Nerven- und Geisteskrankheiten usw. handelt, forscht man natürlich nach rückwärts, sonst aber eben nicht. Der praktische A'zt erfährtalso nur relativ selten etwas über eine etwaige latente Krankheitsanlage und erbliche Belastung, wenn nicht dazu ein besonderer Grund a uffordert. Und wie leicht kann selbst dann nach Jahren der Arzt das einzelne vergessen, wenn er nicht sofortige Notizen darüber sich gemacht hatte. Voraussetzung wäre freilich immer noch, daß die Leute etwas aussagen können. Die meisten sind aber bezüglich ihrer Familienverhältnisse des näheren wenig zu Hause und zwar nicht nur die Ungebildeten. Ich vermag z. B. über die körperlichen und geistigen Vorzüge und Nachteile, ebensowenig über Krankheiten usw. meiner beiden Großeltern von beiden Seiten so gut wie nichts zu berichten. Und nun gehe man erst unter das Volk hinaus! Da wissen die Leute oft nicht einmal, wann der Vater, die Mutter, die Geschwister gestorben sind und woran, ob ein Selbstmord vorgekommen ist usf. Daß endlich über die Trunksucht hier ganz eigene Ansichten herrschen, ist ja bekannt. Das gilt aber auch von den Gebildeten, fa von den Ärzten, je nachdem sie abstinent sind oder nicht.

Es wäre freilich richtiger, statt Rausch ,,akute" und statt Trunksucht ,chronische Alkoholvergiftung“ zu sagen, wenn man nur erst wüßte, von wann ab man von „Vergiftung" reden darf. Bekanntlich kann jedes Ingestum in einer gewissen Quantität giftig wirken. Wenn nun jemand z. B. ein Schnitt Lagerbier trinkt, so werden wohl selbst die strengsten Abstinenzler dies nicht ohne weiteres als „Rausch“ oder „,akute Alkoholvergiftung" hinstellen wollen. Besser ist dagegen der von Fels gebrauchte Ausdruck ,unter Alkoholwirkung stehend" auch

Z. f. d. g. Neur. u. Psych. O. XVII. 
hier anwendbar. Wenn ich also in meiner früheren Arbeit sagte, man müsse den „Rausch" definieren, so gilt dies natürlich auch von der „,akuten Alkoholvergiftung“. Man muß also verlangen, daß die wirklich genosseneAlkoholmenge festgelegt sei, und es nachher dem subjektiven Beurteilen überlassen, ob man diese Menge dann als keimschädigend ansehen will oder nicht, wobei nicht zu vergessen ist, daß der Schaden bei gleicher Menge individuell sicher verschieden ausfallen wird.

Wir sahen oben, daß auch Holitscher die Untersuchung Bezzolas nicht gelten läßt. Schon vorher kannte man die Rauschzeugungen zu Festzeiten. Man sprach daher auch z. B. von „Hochzeitskindern“. Ich habe letzteres hauptsächlich aus dem Grunde abgelehnt, weil für die Tatsache, daß das erste Kind der Ehe gewöhnlich oder mindestens sehr häufig körperlich oder geistig etwas minderwertig ist, ganz andere Momente verantwortlich sind, als gerade eventuell der Alkohol. Daß aber der erste Beischlaf der Hochzeitsnacht gar nicht so abnorm selten befruchtet, was. Fels leugnet, ersieht man aus den Geburtsstatistiken, obgleich das Maximum der Erstgeburten nach dem zweiten Jahre der Ehe fällt. Úbrigens könnte man unter „Hochzeitskindern“ vielleicht auch noch diejenigen Kinder verstehen, die von den Hochzeitsgästen eventuell in der auf das Fest folgenden Nacht gezeugt wurden. Und es scheint fast, als ob von den Gästen im allgemeinen mehr getrunken würde, als vom Brautpaare, das sich immerhin zusammennimmt. Man müßte also auch einmal nach dieser Richtung hin nachforschen. Das gleiche bezieht sich auf die Teilnehmer an Diners, Zweckessen usf., wobei oft des Guten zu viel getan wird. Als ich vor längerer Zeit mit einem Kollegen über Rauschzeugung sprach, erzählte er mir, er sei sicher, daß das unter seinen zum Teil hochbegabten Kindern geistig am wenigsten regsame, jetzt ein Mädchen von 23 Jahren, in einer Nacht gezeugt worden wäre, als er von einer feuchtfröhlichen Gesellschaft bezecht nach Hause gekommen sei. Ein solcher Zusammenhang ist auch mir wahrscheinlich, da er meine Arbeiten kannte, und als Mediziner die zu berücksichtigenden Momente wohl wußte. Freilich lag die Sache weit zurück und da könnte er wohl manches vergessen haben, vor allem ob es sich um einen einzigen Coitus handelte, dem keiner voranging oder folgte.

Holitscher, Fels und wohl auch Hoppe behaupten nun, daß bei Festzeiten, Mostfesten usw. mehr sonst solide Leute sich betrinken, als Säufer, die solche Gelegenheiten zum Rausche gar nicht aufzusuchen brauchten. Mit anderen bin ich der entgegengesetzten Ansicht, da es mir scheint, als ob geradePotatoren solche Gelegenheiten nicht unbenutzt vorübergehen lassen, da sie zumeist Geselligkeit lieben und sich ein Extraräuschchen antrinken, was bei ihnen durchaus nicht jeden Tag 
zu geschehen braucht. Ich bin überzeugt, daß z. B. zu den bayerischen Keller- oder Mostfesten ein Gutteil Trinker und Psychopathen aller Art, die sich zum Teil schnell berauschen, hinzudrängt. Nur eine besonders darauf gerichtete Spezialuntersuchung könnte die Meinungsdifferenz hier schlichten. Sie wäre deshalb wichtig, weil sie den Bezzolaschen Massenuntersuchungen, wenn sie in meinem Sinne ausfielen, eine weitere Stütze entziehen würde.

Es spitzt sich nun alles schließlich auf die Fragen zu: 1. Wird das Blut beim Rausche (resp. akuter Alkoholvergiftung) mit Alkohol sehr überschwemmt, speziell in den Hodengefäßen und in der Hodenflüssigkeit ; 2 . bei welcher Menge desselben tritt eine Keimschädigung ein, und 3. wie kommt diese Keimschädigung zustande? Eine Nebenfrage wäre dann noch die, ob die gleiche Alkoholmenge die Spermatozoen mehr angreift als das Eichen. Auf diese Fragen wissen wir nur wenig zu antworten. Experimente an Pflanzen- und Tiersamen im Laboratorium besagen deshalb nicht viel, weil die Spermien hier isoliert, in einem fremden Milieu sind. Außerdem sind sie zum Teil widersprechend ${ }^{1}$ ). Daß der Alkohol bis in die Keimdrüsen dringt, ist sicher, da dieselben vom allgemeinen Blutkreislaufe ja nicht ausgeschlossen sind. Aber zerstört werden diese Drüsen durch Alkohol doch nur allmählich, und davon kann natürlich beim gelegentlichen Rausche keine Rede sein.

Ich hatte seinerzeit auf die mannigfachen Schwierigkeiten hingewiesen, die dem Einwirken des Alkohols auf das Sperma und Eichen bei so geringen Dosen entgegenstehen, Bemerkungen, die mit anderen mehr oder minder dazugehörigen Holitscher zum Teil kaum bekannt sein konnten und auch sicher nicht interesselos waren. Ich hatte speziell gesagt, daß der Einfluß des Alkohols in letzter Instanz bloß durch Experimente und mikrochemische und mikroskopische Untersuchungen entschieden werden könne. Nach Schweisshei mer ${ }^{2}$ ) findet sich nun die größte Menge Alkohol im Blute Betrunkener und zwar soll beim nicht gewohnten Organismus die Alkoholkonzentration eine höhere sein und sich länger so halten, als beim gewöhnten. Der Zustand der Trunkenheit verlaufe parallel dem Steigen und Fallen der Alkoholkonzentration. Angenommen, daß dies wirklich sich so verhält, so käme für uns doch hauptsächlich nur der Alkoholgehalt des Blutes in den Keimdrüsen und der Spermaflüssigkeit in Frage. Nun sollen die Keimdrüsen gewöhnlich spezielle anatomische Schutzkräfte gegen

1) Ich erwähne hier nochmals die Experimente Iwanows, der die Spermien von Säugetieren in sehr hohem Grade gegen Alkohol refraktär fand und damit leicht geborene, wohl entwickelte und gesunde Nachkommen erzeugte, im Gegensatz zum Verhalten bei Einführen von Alkohol in den Organismus.

2) Schweissheimer, Alkoholgehalt des Blutes. Deutsches Archiv f. klin. Med. 109, 1913; Ref. in Deutsche med. Wochenschr. 1913, S. 521 und Münchner med. Wochenblatt 1913 , S. 1105. 
Vergiftungen haben. Man müßte auch ferner wissen, ob die Keimdrüsen ebenso blutreich sind, wie andere Drüsen. Den Alkoholgehalt derselben dürfte man am sichersten durch Punktion der Hoden (resp. besser noch der Samenblasen, siehe später) in Lokalanästhesie bei Betrunkenen feststellen, wobei Potatoren natürlich auszuschließen wären. Diese harmlose Operation ist wohl noch nicht zu diesem Zwecke versucht worden.

Wegen der mikrochemischen Frage wandte ich mich an eine der größten Autoritäten auf dem Gebiete der Biochemie, an Prof. Carl Oppenheimer in Berlin. Am 1. März 1913 schrieb er mir folgendes:

„.... Soweit mir bekannt ist, sind Organe von an akutem Rausch gestorbenen Menschen nur außerordentlich selten untersucht worden und man hat dabei gefunden, daß sich nicht ganz $1 / 2 \%$ Alkohol im Blute vorfindet, während sehr lipoidreiche Organe, insbesondere das Gehirn, etwas reicher daran sind. Es wäre also auch möglich, daß sich die ja ebenfalls sehr lipoidreichen Spermatozoen ebenfalls mit Alkohol anreichern, doch dürfte es sich in diesem Falle jedenfalls um äußerst geringe Mengen handeln. Die Schwierigkeit betrifft den sicheren Nachweis des Alkohols. Dieser läßt sich unter keinen Umständen, wie Sie andeuten, mikrochemisch führen, sondern ist nur durch die Jodoformreaktion mit einer großen Unsicherheit, sicher hingegen nur durch exakte Darstellung des reinen Alkohols zu führen, ein Verfahren, das natürlich in der Analyse von Samenflüssigkeit u. dgl., wo es sich um sehr geringe Mengen handelt, kaum durchzuführen sein wird. ..."

Wir sehen also daraus, daß in den Organen an akutem Rausch gestorbener Menschen nicht ganz $1 / 2 \%$ Alkohol sich im Blute vorfindet; wieviel im Hoden, ist nicht gesagt. Von einem solchen Grade von Rausch ist aber bei unserem Thema nicht die Rede, denn dann hört überhaupt die Kohabitationsfähigkeit auf. Diesen Angaben gemäß dürfte also wohl beim gewöhnlichen Angeheitertsein die Konzentration des Alkohols im Blute (ob auch soviel im Hoden und in dem Sperma, ist noch ungewiß) noch weit darunter sinken, - als Höchstwert des Alkohols im Blute von Betrunkenen fand Schweissheimer 2,266 pro mille - also eine so minimale Menge, daß an ihren schädigenden Einfluß auf gesunde und erwachsene Spermien usw. kaum zu denken ist ${ }^{1}$ ). Und sollte sie noch unreife, junge Spermatozoen mehr angreifen, was wohl möglich ist, so steht nach Experimenten am Tier zu erwarten, daß im Kampfe um den Eintritt in die Mikropyle des Eichens die ausgewachsenen Exemplare Sieger bleiben, zumal solche normalerweise bei der Ejakulation hauptsächlich vertreten sind. Nach Weissenberg ${ }^{2}$ ) befindet sich nun aber ,,sehr wahrscheinlich“ das Sperma kurz

1) Das würde auch für den ersten Fall Holitschers gelten. Die von Holitscher und Fels hier berechnete Alkoholmenge im Blute ist nach obigen Angaben $O$ ppenheimers sicher zu hoch gegriffen.

2) Weissenberg, Anatomie und Physiologie der Genitalapparate des Mannes. In: Moll, Handbuch der Sexualwissenschaften usw. Leipzig 1912, S. 43. 
vor der Ejaculation in der Samenampulle oder gar in der Samenblase, d. h. nicht mehr im eigentlichen Hoden, also in Höhlen mit einer relativ blutarmen Wand, so daß hier noch weniger Alkohol eintreten dürfte als im Hoden selbst und das noch um so weniger, als das Samenblasensekret sehr dicklich ist und der Diffusion des Alkohols gewiß so noch mehr Schwierigkeit bereitet. Ihr Inhalt dürfte daher so gut wie alkoholfrei bleiben. Aber auch im Hoden selbst ist der Gehalt an Alkohol sicher ein nur geringer, zumal natürliche Schutzvorrichtungen dagegen für gewöhnlich vorliegen. Prof. Fürbringer in Berlin, der ja gerade sehr viel in Mikroskopie und Chemie des Spermas gearbeitet hat, schreibt mir am 24. Februar 1913 u. a. auch: „Daß man bei der in Betracht kommenden mehr als homöopathischen Menge den Alkohol im Bereich der Spermien mikrochemisch nachzuweisen vermöchte, bedeutet für mich nur eine entfernte Möglichkeit." Ja Bayerthal'1), der sonst für die Rauschzeugung eintritt, meint sogar, daß nicht bei jedem Rausche Alkohol in den Samen gelange ${ }^{2}$ ) und zwar wegen Vorhandenseins anatomischer Schutzvorrichtungen, die freilich manchmal mangelhaft seien. Dafür spräche u. a. die gute Beschaffenheit vieler Hochzeitskinder. Eher wäre schon denkbar, wie dies Hoppe seinerzeit geäußert hat, daß durch solche geringe Mengen von Alkohol die unreifen Spermien im Hoden geschädigt würden und so durch einen späteren Beischlaf eine Minderwertigkeit der Frucht zuwege käme.

Interessant war mir die Angabe 0 p penheimers, daß zurzeit der Alkohol sich mikrochemisch nicht nachweisen läßt, und das scheint auch Fürbringer anzunehmen. Das würde wohl auch mit der von Schweissheimer (l. c.) zur Bestimmung von kleinen Alkoholmengen empfohlenen Methode von Nicloux unmöglich sein. Damit ist aber freilich nicht gesagt, daß ein solcher mikrochemischer Nachweis später nicht doch noch gelingen wird, ebenso wie auch dann vielleicht ein mikroskopischer Befund. Fels meint, es gäbe noch eine Möglichkeit von Strukturveränderung der Samenzellen, nämlich eine indirekte, die durch veränderten Chemismus der Samenflüssigkeit z. B. gesetzt werden und so schaden könnte, ohne daß Alkohol also selbst in den Spermien nachzuweisen wäre. Man müßte freilich zunächst hier beweisen, daß durch so minimale Dosen Alkohol der Chemismus der Samenflüssigkeit sich wirklich wesentlich ändert. Auch dann wird man sich nur schwer vorstellen können, wie in einer so kurzen Zeit der Zirkulation die Er-

1) Bayerthal, Jahresbericht der schulärztlichen Tätigkeit an der städtischen Hilfsschule in Worms. (Schuljahr 1908/09.)

$\left.{ }^{2}\right)$ Ich bemerke, daß manche Psychopathen zwar eine große Sucht zum Trinken zeigen, aber sehr leicht sich berauschen, oft nach lächerlich kleinen Alkoholmengen, so daß dann hier speziell sicher nur ganz wenig Alkohol in das Blut kommt und also in die Hoden. 
nährungsverhältnisse der Spermazellen sich so verschlechtert und Strukturveränderungen gesetzt haben sollen; eher schon könnte durch veränderten Chemismus der Umgebung die Lebhaftigkeit der Bewegungen der Spermien nachlassen.

Besonders anregend waren mir aber folgende briefliche Auslassungen von Prof. Abderhalden in Halle vom 24. Februar 1913, die zu verschiedenen Bemerkungen mir Anlaß geben.

„Ich habe selbst vor mehreren Jahren versucht, Hunde in akuten Rauschzustand zu versetzen. Ioh gab den Alkohol per Schlundsonde. Ich konnte leider nie einen richtigen Rauschzustand erhalten. Die Tiere taumelten, fielen dann hin und waren schließlich ganz soporös. An einen Coitus war nicht zu denken! Es wäre denkbar, daß man ein akut vergiftetes Weibchen befruchten lassen kann von einem nicht alkoholisierten Männchen. Wollte man Weibchen und Männchen alkoholisieren, dann müßte man die künstliche Befruchtung versuchen. Es ist dies der einzige Weg! - Es gibt Methoden, um sehr geringe Mengen von Alkohol aufzufinden. Niclo $\mathrm{u} x$ hat den Alkohol im Blute und auch im Hoden nachgewiesen. Ich persönlich kann mir schon vorstellen, daß bei akutem Rauschzustand die Geschlechtszellen momentan geschädigt sind. Ob alle Zellen gleichmäßig affiziert sind, ist schwer ohne Experiment auszusagen. Man müßte auch hier mit künstlicher Befruchtung arbeiten und z. B. Seeigeleier und Samen in eine so verdünnte Alkohollösung bringen, wie sie dem Gehalt des Blutes an Alkohol im Rauschzustand entspricht. - Es fehlt, wohin man auch sieht, in der wissenschaftlichen Alkoholfrage an Grundlagen. Schon seit Jahren werde ich vom Mäßigkeitsverein in Berlin aufgefordert, Vorträge bei den wissenschaftlichen Kursen zu halten. Ich sage immer ab, weil ich nicht in der Lage bin, irgendein Problem über die physiologischen Wirkungen des Alkohols auf Grund der vorliegenden Versuche in positivem Sinne zu behandeln. Bestimmte Schlüsse lassen sich nirgends ziehen. In Amerika soll am Roc kefeller-Institut eine Abteilung zur Erforschung der Alkoholwirkung aufgetan werden. Ich freue mich, daß an einer so neutralen Stätte derartige Studien aufgenommen werden. Diese Herren werden für jede Anregung sehr dankbar sein...."

Wir sehen daraus zunächst, daß Abderhalden erfolglos Hunde in einen richtigen Rauschzustand zu versetzen suchte und solche hohe Mengen von Alkohol wird ein bloß Bezechter sicher nicht zu sich nehmen. Schon früher (in meiner zweiten Arbeit) hatte ich auseinandergesetzt, daß das plötzliche Einführen von Alkohol absolut dem ,sich Betrinken" nicht an die Seite gesetzt werden darf, das meist im Verlaufe mehrerer Stunden vor sich geht. Man müßte, um möglichst gleiche Bedingungen zu setzen, soviel Alkohol dem Hunde irgendwie beibringen, daß bei ihm das Blut eine ähnliche Konzentration davon erhält wie beim Menschen. Man könnte dann von den psychischen Zeichen, die beim Menschen natürlich viel früher und differenzierter auftreten müssen, ganz absehen und hätte als Vergleich nur den gleichen Alkoholgehalt des Blutes oder besser gesagt, der Hodenflüssigkeit. Dabei wird noch vorausgesetzt, daß der Alkohol auf den Samen von Mensch und Tier gleich giftig einwirke. Schließlich hätte man den vorgeschlagenen Begattungsversuch oder die künstliche Befruchtung vorzunehmen. Wohl 
kann sich ferner Abderhalden ,vorstellen“, daß die akuten Rauschzustände die Geschlechtszellen momentan schädigen können. Ob dabei letztere gleichmäßig betroffen würden, sei schwer ohne Experiment zu entscheiden und er schlägt hierfür die künstliche Befruchtung von alkoholisierten Keimzellen in der Schale vor, was ich für wenig beweisend halte, da hier die Zellen aus ihrem natürlichen Verbande ganz losgelöst sind.

Sehr wichtig aber ist endlich der Satz Abderhaldens, daß es, wohin man sähe, in der wissenschaftlichen Alkoholfrage an den Grundlagen fehle, daher bestimmte Schlüsse sich zurzeit nicht ziehen ließen. Dies erscheint um so gewichtiger, als Abderhalden selbst Abstinent ist und die gesamte alkoholische Literatur beherrscht, wie sicher nur wenige, und trotzdem er aus obigem Grunde verschmäht, propagandistisch zu wirken. Es ist mit Freude zu begrüßen, daß das Rockefeller - Institut in New York sich demnächst wissenschaftlich mit der Alkoholfrage abgeben will. Ich werde nicht verfehlen, die dortigen Gelehrten, die hoffentlich sine ira et studio arbeiten werden, speziell auch auf das Problem der Rauschzeugung hinzuweisen und zu bitten, durch genaue Enqueten und besonders durch Experimente usw. unsere Frage der Lösung vielleicht bald näher zu bringen. Zuvor werde ich aber auch, wie hier so in der Deutschen Medizinischen Wochenschrift - in aller Kürze natürlich - die praktischen Ärzte für das vorliegende Problem zu interessieren suchen und sie durch genaue Berichterstattung aus ihrer Erfahrung zur Mitarbeit auffordern. Mit bloßen Eindrücken ist uns allerdings hier nicht geholfen. Die Sache ist ja nicht nur an sich schon wissenschaftlich hochinteressant, sondern kann auch von großer Tragweite sein.

Ich glaube für mich das Verdienst in Anspruch nehmen zu dürfen, die ganze Frage der Rauschzeugung, die wirklich zum reinen Schlagwort herabgesunken war, in wissenschaftlichere Bahnen geleitet und gezeigt zu haben, daß man auch hier ganz strenge Forderungen stellen muß. Weiterhin hat sich im Anschluß an meine Arbeiten gezeigt, daß nur die 3 Fälle Holitschers als solche einer nachgewiesenen Rauschzeugung sich halten lassen, als vierter Fall etwa noch der von mir oben mitgeteilte eines Kollegen. Seit 1908 bis jetzt also nur höchstens vier Beispiele! Das dürfte doch auf die große Seltenheit eines solchen Ereignisses hinweisen; die Möglichkeit davon habe ich nie bestritten, nur die Häufigkeit. Mitwirkend dagegen wird auch hier öfters der Alkohol verderblich sein können, so z. B. bei an sich schon vererbter psychoxothischer Anlage. Vielleicht wird man noch einige weitere Fälle beibringen ${ }^{1}$ ). Immer ist bei solchen Untersuchungen jedoch bloß von

1) Dabei sind aber möglichst streng die obigen Erfordernisse einzuhalten. Dies fehlt z. B. in der mir am 1. Mai von einem bekannten psychiatrischen 
Einzelbeobachtungen auszugehen. Diese sowie allerlei Experimente mit vielleicht künftigen mikrochemischen und mikroskopischen Untersuchungen werden uns weiter führen, nie aber Massenuntersuchungen à la Bezzola und Bayerthal mit den vielen naturgemäß ihnen anhaftenden Fehlerquellen. Massenuntersuchungen sind daher am besten beiseite zu setzen, womit natürlich nicht gesagt sein soll, daß unter ihnen sich nicht doch vielleicht noch manche Fälle von wirklicher Rauschzeugung befinden, nur sind sie hier eben schwer herauszuschälen.

Es ist schon viel gewonnen, wenn jetzt die Autoren, aufmerksam gemacht, mit dem Worte „Rauschzeugung“" vorsichtiger geworden sind. Und in der Tat findet sich dies Schlagwort in den neueren Arbeiten viel seltener als früher. $\mathrm{Zu}$ den schwierigsten Aufgaben einer jeden Wissenschaft gehört jederzeit die nach der Kausalität. Wir Psychiater insbesondere wissen dies am besten. Hier kann man nicht kritisch genug verfahren und muß die Tatsachen von allen Seiten betrachten. Man darf ferner nicht jedes neue Faktum auf Grund von Experimenten usw. schon ohne weiteres als Wahrheit proklamieren, sondern muß dasselbe erst vielfach nachprüfen, bevor es in das wissenschaftliche Rüstzeug eingefügt werden darf. Vor allem aber soll man nicht voreingenommen an eine Aufgabe herantreten, sonst trübt sich nur zu leicht der Forscherblick. Ein einziger Affekt sollte uns alle beim wissenschaftlichen Arbeiten beseelen: der Wahrheit zu dienen und darin die höchste Befriedigung $\mathrm{zu}$ finden.

Hochschullehrer des Auslandes gewordenen Mitteilung. Eine Frau war zweimal verheiratet gewesen und die zwei Knaben der ersten Ehe waren ganz normal und blieben es auch im späteren Leben. Vom zweiten Mann hatte sie ein Kind, jetzt $13 \mathrm{Jahr}$ alt. Der Mann hatte zur Zeit der Empfängnis viel getrunken. Das Kind zeigt körperliche und geistige Entartungszeichen, war von klein auf unverbesserlicher Lügner, Dieb, Faulenzer, ward von der Schule fortgeschickt, hielt nirgends aus, zeigte fugues usw. Man sieht, daß in diesem kurzen Berichte noch manches Erforderliche fehlt, der psychische Zustand des Vaters und der Mutter ist nicht beschrieben, auch soll der Mann zur Zeit der Konzeption viel getrunken haben, d. h. doch wohl nicht nur einmal bezecht gewesen sein, sondern sicher eine Art von Trinker usw. Immerhin gebe ich zu, daß dieser Fall mindestens der Rauschzeugung verdächtig erscheint. 